\title{
A new 5d description of $6 d$ D-type minimal conformal matter
}

\author{
Hirotaka Hayashi, ${ }^{a}$ Sung-Soo Kim, ${ }^{b}$ Kimyeong Lee, ${ }^{b}$ Masato Taki ${ }^{c}$ and Futoshi Yagi ${ }^{b}$ \\ ${ }^{a}$ Instituto de Física Teórica UAM/CSIC, \\ Cantoblanco, 28049 Madrid, Spain \\ ${ }^{b}$ Korea Institute for Advanced Study (KIAS), \\ 85 Hoegiro Dongdaemun-gu, 130-722, Seoul, Korea \\ ${ }^{c}$ iTHES Research Group, RIKEN, \\ Saitama 351-0198, Japan \\ E-mail: h.hayashi@csic.es, sungsoo.kim@kias.re.kr, klee@kias.re.kr, \\ taki@riken.jp, fyagi@kias.re.kr
}

ABStRact: We propose a new 5 d description of the circle-compactified $6 \mathrm{~d}\left(D_{N+4}, D_{N+4}\right)$ minimal conformal matter theory which can be approached by the $6 \mathrm{~d} \mathcal{N}=(1,0) \operatorname{Sp}(N)$ gauge theory with $N_{f}=2 N+8$ flavors and one tensor multiplet. Compactifying the brane set-up for the $6 \mathrm{~d}$ theory, we arrive at a 5 -brane Tao diagram for $5 \mathrm{~d} \mathcal{N}=1 \mathrm{SU}(N+2)$ theory of the vanishing Chern-Simons level with $2 N+8$ flavors. We conjecture that the $6 \mathrm{~d}$ theory is recovered as the UV fixed point of this $5 \mathrm{~d}$ theory. We show that the global symmetry of this $5 \mathrm{~d}$ theory is $\mathrm{SO}(4 N+16)$ identical to that of the $6 \mathrm{~d}$ theory by analyzing the 7-brane monodromy. By using the Tao diagram, we also find the instanton fugacity is exactly given by the circle radius. By decoupling flavors in this $5 \mathrm{~d}$ theory, one can obtain all the $5 \mathrm{~d} \mathrm{SU}(N+2)$ gauge theories of various Chern-Simons levels and corresponding enhanced global symmetries at the 5d UV fixed point.

Keywords: Brane Dynamics in Gauge Theories, Field Theories in Higher Dimensions, Global Symmetries

ArXiv EPrint: 1505.04439 


\section{Contents}

1 Introduction and conclusion $\quad 1$

2 From 6d to 5d 3

2.1 Evidence 1: O-plane description 4

2.2 Evidence 2: $\hat{D}$-type Dynkin quiver 6

3 Global symmetry of SU(n) gauge theories 10

\section{Introduction and conclusion}

Recently there has been an intense investigation of the $6 \mathrm{~d} \mathcal{N}=(1,0)$ superconformal field theories (SCFTs) by using the F-theory compactification on elliptically fibered Calabi-Yau spaces and the various brane pictures, which includes their strings [1-6], classifications [712], compactifications [13, 14], and anomaly polynomials as well as RG flows [15-18] (also see [19-21]). Typical examples are those on the M5 branes near the $E_{8}$ symmetric M9 wall or on the transverse $A D E$ type singularities or both. Interestingly, the 6d SCFT on a single M5-brane probing the $E_{8}$ symmetric M9-brane $[22,23]$ can be also realized as the theory on a single M5-brane probing a $D_{4}$ singularity, namely the $6 \mathrm{~d}\left(D_{4}, D_{4}\right)$ minimal conformal matter $[7,9,13]$. It is also known that this $6 \mathrm{~d}$ theory arises as the theory at the UV fixed point of the $5 \mathrm{~d} \mathrm{SU}(2)$ gauge theory with 8 flavors [24, 25]. Its coupling constant is proportional to the size of the compact circle and the strong coupling limit of the $5 \mathrm{~d}$ theory would be the $6 \mathrm{~d}$ theory. By decoupling flavors one by one from the $5 \mathrm{~d}$ theory, one can realize $5 \mathrm{~d} \mathrm{SU}(2)$ gauge theories with $N_{f}<8$ flavors which have the $5 \mathrm{~d}$ strongly coupled fixed point with enhanced $E_{N_{f}+1}$ global symmetry [24-26]. There are old brane diagram arguments [27], recent index calculations [28, 29], and more recent related arguments to support this symmetry enhancement [30-46].

The $5 \mathrm{~d} \mathrm{SU}(2)$ gauge theories have yet another realization as a $(p, q) 5$-brane web diagram. The $\mathrm{SU}(2)$ gauge theories with $0 \leq N_{f} \leq 7$ flavors have a usual web diagram realization $[27,47,48]$ including diagrams suggested in $[42,49]$. However, when the number of the flavors reaches eight, we need to consider a new class of the web diagram, so-called Tao web diagram introduced in [50], which is given by 5-branes that infinitely expand in a spiral configuration. The appearance of the spiral is interpreted as the $S^{1}$ for the 6 th dimension. An important characteristic of the Tao diagram is that its period of the spiral rotation is identified with the instanton factor of the theory.

From the point of view of the Tao diagram [50], the rank of the gauge group is not limited and various Tao diagrams are possible including quiver gauge theories, which may reflect close connection of Tao diagrams to various 6d SCFTs. However, the relation between the classification of $6 \mathrm{~d}$ SCFTs and the $5 \mathrm{~d}$ theories realized by the Tao diagrams 
was not clear. In this paper, we argue that the $\mathrm{SU}(n)$ type Tao diagram can have a definite relation to $6 \mathrm{~d}$ SCFTs. We identify its $6 \mathrm{~d}$ origin and claim that it is the generalization of the correspondence between the $5 \mathrm{~d} \mathrm{SU}(2)$ gauge theory with 8 flavors and the $\left(D_{4}, D_{4}\right)$ minimal conformal matter on $S^{1}$. Our main conjecture is that $5 \mathrm{~d} \mathrm{SU}(N+2)$ gauge theory with $N_{f}=2 N+8$ flavors with the vanishing Chern-Simons (CS) level has the 6d UV fixed point which is the $\left(D_{N+4}, D_{N+4}\right)$ minimal conformal matter. We also argue that the global symmetry at the fixed point is enhanced to $\mathrm{SO}(4 N+16)$.

Moreover, starting from the $5 \mathrm{~d} \mathrm{SU}(N+2)$ gauge theory with $N_{f}=2 N+8$ and decoupling flavors one by one, it is again possible to construct all the $5 \mathrm{~d} \mathrm{SU}(N+2)$ gauge theories with the various CS level which have the $5 \mathrm{~d}$ UV fixed point. The Tao diagram then reduces to a standard web diagram of $(p, q)$ 5-branes. Bergman and Zafrir have already conjectured the existence of the UV fixed points for $\mathrm{SU}(N+2)$ theories with $N_{f} \leq 2 N+7$ in $[40]$.

In the following, we summarize our idea and results. In section 2 , we consider two paths to support our conjecture. One way is to use the brane set up [51,52] for the $6 \mathrm{~d}$ $\operatorname{Sp}(N)$ gauge theory with $N_{f}=2 N+8$ fundamental hypermultiplets and a single tensor multiplet coupled to cancel the gauge anomaly $[53,54]$ by the Green-Schwarz mechanism, ${ }^{1}$ which corresponds to the $\left(D_{N+4}, D_{N+4}\right)$ minimal conformal matter in the tensor branch. After circle compactification with Wilson line breaking $\operatorname{Sp}(N)$ to $\mathrm{U}(N)$, we explain how we obtain $5 \mathrm{~d} \mathrm{SU}(N+2)$ gauge theory with $2 N+8$ fundamental hypermultiplets by brane dynamics. Another way is to use the description as the worldvolume theory on a single M5-brane probing the orbifold singularity $\mathbb{C}^{2} / \Gamma_{D_{N+4}}$. Compactifying on $S^{1}$ the M5-brane would yield type IIA setup of a single D4-brane on the same singularity, and the resulting $5 \mathrm{~d}$ theory is well-known to be associated with the affine D-type Dynkin quiver $[9,56]$. Here we propose a 5-brane web realization of this quiver theory and the S-dual of this is precisely the $\mathrm{SU}(N+2)$ gauge theory with $2 N+8$ flavors. We also show that the expected relation between the gauge couplings and the compactified radius is reproduced via Tao diagram.

In section 3 , we systematically study the global symmetry enhancement of the $5 \mathrm{~d} \mathrm{SU}(n)$ theory with $N_{f} \leq 2 n+4$ flavors. $^{2}$ We start from the brane web diagram for the $5 \mathrm{~d} \mathrm{SU}(n)$ theory with $2 n+4$ flavors and remove the flavor multiplets one by one by making its mass infinite. Each removal generates the half integer Chern-Simons level whose sign depends on the sign of the mass term, leading to a class of $5 \mathrm{~d} \mathrm{SU}(n)_{\kappa}$ theories with $N_{f}$ flavors ${ }^{3}$ with constraint $N_{f}+2|\kappa| \leq 2 n+4$. These $5 \mathrm{~d} \mathrm{SU}(n)_{\kappa}$ theories with $N_{f}$ flavors with constraint $N_{f}+2|\kappa| \leq 2 n+4$ have classically $\mathrm{SU}\left(2 N_{f}\right) \times \mathrm{U}(1)_{B} \times \mathrm{U}(1)_{\text {I }}$ global symmetry where $\mathrm{U}(1)_{B}$ is the baryonic part of the flavor symmetry and $\mathrm{U}(1)_{\mathrm{I}}$ is the topological symmetry arising from the instanton numbers. These theories have an enhanced symmetry at the UV fixed point which is a subgroup of $\mathrm{SO}(4 n+8)$. We calculate enhanced symmetry for all of the

\footnotetext{
${ }^{1}$ The $6 \mathrm{~d}$ theories with gauge group $\mathrm{SU}(2)$ and $N_{f}$ fundamental matter wit a tensor multiplet has an additional constraint on $N_{f}$ by the global anomaly cancellation due to $\pi_{6}(\mathrm{SU}(2))=\mathbb{Z}_{12}$ [55]. The acceptable number of flavors for the $\mathrm{SU}(2)$ gauge case is $N_{f}=4,10,16$. The $6 \mathrm{~d}$ counter part for our $5 \mathrm{~d} \mathrm{SU}(3)$ theory with $N_{f}=10$ is this $6 \mathrm{~d} \operatorname{Sp}(1)$ theory with $N_{f}=10$.

${ }^{2}$ We use the notation $n=N+2$ for convenience.

${ }^{3}$ We use the notation that $\mathrm{SU}(n)_{\kappa}$ represents that $\mathrm{SU}(n)$ gauge theory of Chern-Simons level $\kappa$.
} 
$\mathrm{SU}(n)_{\kappa}$ theories with flavors $2 n \leq N_{f} \leq 2 n+4$ and of the $\mathrm{SU}(3)_{\kappa}$ theories with flavors $N_{f}+2|\kappa| \leq 10$. Especially, we find that the $\mathrm{SU}(n)_{\frac{1}{2}}$ theory with $N_{f}=2 n+3$ flavors has $\mathrm{SO}(4 n+8)$ global symmetry which is identical to that of the $6 \mathrm{~d}$ theory. This is a generalization of the $5 \mathrm{~d} \mathrm{SU}(2)$ theory with $N_{f}=7$ flavors having an enhanced $E_{8}$ global symmetry. Our result in section 3 is consistent with a few cases found recently in [34, 40], where the authors have studied the enhanced global symmetry for $\mathrm{SU}(n)_{n}$ with $N_{f}=0$, the most cases of $\mathrm{SU}(3)_{\kappa}$ with $N_{f} \leq 8$. We enclose all cases for the completeness.

As summarized above, we provide several evidences for our conjecture in this paper. It would be certainly desirable to find more supports to establish the conjecture. One way is to calculate the index function with very careful treatment of instanton computation from the $5 \mathrm{~d}$ point of view and show that the global symmetry is enhanced to $\mathrm{SO}(4 N+16)$ [57]. A similar calculation has been done for the $\mathrm{SU}(2)$ case with $E_{8}$ global symmetry $[4,36]$. As done in [50], it is possible to compute the partition function applying the topological vertex method to the Tao diagram [57]. Another way is to start from the $6 \mathrm{~d}$ point of view. With the introduction of the $\operatorname{Sp}(N)$ Wilson line, together with the contribution from the tensor multiplet, the elliptic genus calculation of instanton strings may lead to the $5 \mathrm{~d} \mathrm{SU}(N+2)$ gauge symmetry in the Coulomb phase [57]. Our observation for the relation between the $6 \mathrm{~d}$ and $5 \mathrm{~d}$ supersymmetric theories may lead to further examples and insights.

Note added. We here note that our results have some overlap with [58], which appeared in arXiv on the same day.

\section{From $6 d$ to $5 d$}

In this section, we justify our main conjecture from two different routes. Our main conjecture is that the $5 \mathrm{~d} \mathrm{SU}(N+2)$ gauge theory with $N_{f}=2 N+8$ flavors and the vanishing CS level has the 6d UV fixed point that is the $\left(D_{N+4}, D_{N+4}\right)$ minimal conformal matter. In other words, a circle compactification of the $\left(D_{N+4}, D_{N+4}\right)$ minimal conformal matter with Wilson line along it gives the the $5 \mathrm{~d} \mathrm{SU}(N+2)$ gauge theory with $N_{f}=2 N+8$ flavors and the zero CS level. Hence, our approach is to start from the D-type minimal conformal matter on $S^{1}$ and then arrive at the $5 \mathrm{~d} \mathrm{SU}(N+2)$ gauge theory with $N_{f}=2 N+8$ flavors and the zero CS level.

In section 2.1, we first go to the tensor branch of the $\left(D_{N+4}, D_{N+4}\right)$ minimal conformal matter. An $S^{1}$ compactification of the system is given by $N$ D6-branes on top of an $\mathrm{O}^{+}{ }_{-}$ plane suspended between two NS5-branes and also semi-infinite $N+4$ D6-banes on top of an $\mathrm{O} 6^{-}$-plane on the left-hand side of the left NS5-brane and the right-hand side of the right NS5-brane in type IIA string theory [9, 51, 52]. The configuration is depicted in the leftmost diagram in figure 1. The resulting theory is the $6 \mathrm{~d} \operatorname{Sp}(N)$ gauge theory with $N_{f}=2 N+8$ flavors and a tensor multiplet. In fact, the same 6 d theory can be also realized by another brane set up in type IIA string theory depicted in the middle diagram in figure 1 . In this case, we have again $2 N$ D6-branes ${ }^{4}$ suspended between NS5-brane and its mirror image through $\mathrm{O}^{-}$. However, in order to realize the $\operatorname{Sp}(N)$ gauge group and

\footnotetext{
${ }^{4}$ The $2 N$ includes the mirror image of D6-branes.
} 

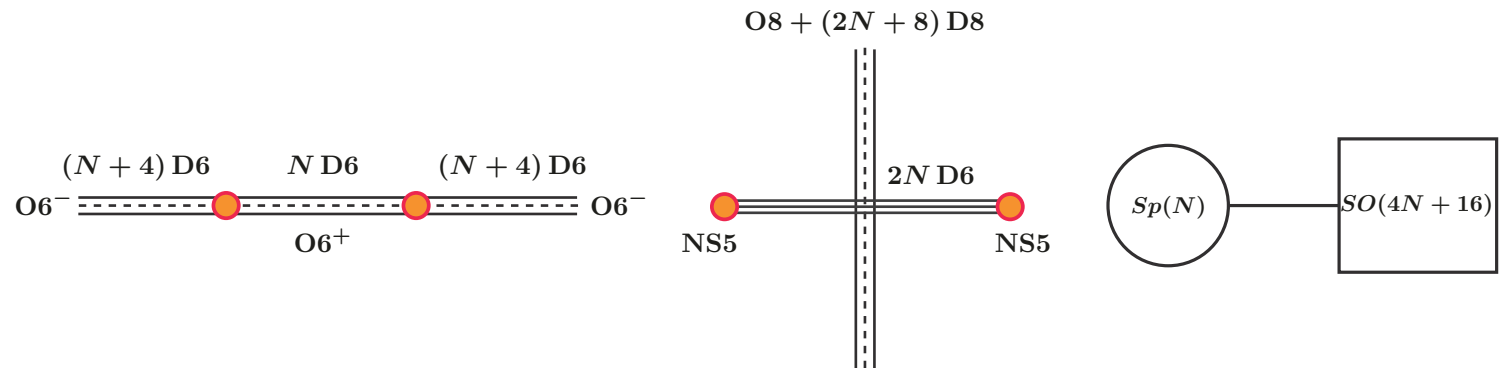

Figure 1. Left: type IIA brane realization of the $\left(D_{N+4}, D_{N+4}\right)$ minimal conformal matter in the tensor branch. Middle: another brane realization of the same $6 \mathrm{~d}$ theory. Right: the quiver diagram of the $6 \mathrm{~d}$ theory.

also flavors, we introduce $2 N+8$ D8-branes on top of an $\mathrm{O}^{-}$-plane $[51,52]$. Then, we again obtain the $6 \mathrm{~d} \operatorname{Sp}(N)$ gauge theory with $N_{f}=2 N+8$ flavors and a tensor multiplet coupled. The quiver diagram (without a tensor multiplet) of the $6 \mathrm{~d}$ theory is depicted in the rightmost diagram in figure 1. This type IIA brane set up is the starting point in section 2.1.

In section 2.2 , we use a different route. We first reduce the $6 \mathrm{~d}\left(D_{N+4}, D_{N+4}\right)$ minimal conformal matter on $S^{1}$ with the Wilson line along it, and go to the $5 \mathrm{~d}$ affine $\hat{D}_{N+4}$ quiver theory. We propose a $(p, q) 5$-brane web diagram which realizes the $5 \mathrm{~d}$ affine $\hat{D}_{N+4}$ quiver theory, and the IIB brane set up is the starting point in section 2.2 .

\subsection{Evidence 1: O-plane description}

We now explain the main conjecture by using the brane description depicted in the middle diagram in figure 1. As discussed before, this $6 \mathrm{~d} \operatorname{Sp}(N)$ theory is known to have the type IIA brane description with $N$ D6 branes suspended between two NS5-branes, with the insertion of $2 N+8 \mathrm{D} 8$ branes and one $\mathrm{O}^{-}$-plane [51, 52]. We compactify one of the direction parallel to all these branes with the Wilson loop. Taking T-dual along this compactified direction, we obtain the type IIB brane setup with $N$ D5 branes, two NS5 branes, $2 N+8 \mathrm{D} 7$ branes and two $\mathrm{O}^{-}$-planes. Due to the Wilson loop, the $N$ D5 branes appear away from both of the two $\mathrm{O}^{-}$-planes, breaking $\operatorname{Sp}(N)$ gauge group to $\mathrm{U}(N)$.

We note that quantum resolution of $\mathrm{O}^{-}$-plane is given by the pair of $[1,1] 7$-brane and $[1,-1] 7$-brane [59]. When we apply this property to the two $\mathrm{O}^{-}-$-planes in this brane setup, we assume that a "5-brane loop" [27] appears, where the original two NS-branes being part of this 5-brane loop and all the 7-branes are inside the 5-brane loop. The $\mathrm{Sp}(1)$ case is depicted in figure 2. Note that the 5-brane charge between the cut created by the $[1,1] 7$-brane and the $[1,-1] 7$-brane should be $(1,0)$, implying that, as a consequence, two more color D5-branes are generated in this process. ${ }^{5}$ Therefore, we obtain $N+2$ D5-branes in total. When we pull out the 7 -branes outside the 5 -brane loop by taking into account the Hanany-Witten transition [60], we obtain the $(p, q)$ 5-brane web, figure 3 , where the

\footnotetext{
${ }^{5} \mathrm{An}$ analogous process is discussed, for example, in [27] relating the type IIA brane setup with an O8plane for the $5 \mathrm{~d} \operatorname{Sp}(1)$ gauge theory to the type $\operatorname{IIB}(p, q)$ brane web description. Contrary to our case, two D5-branes become a part of the 5-brane loop and two more NS5-branes are generated.
} 


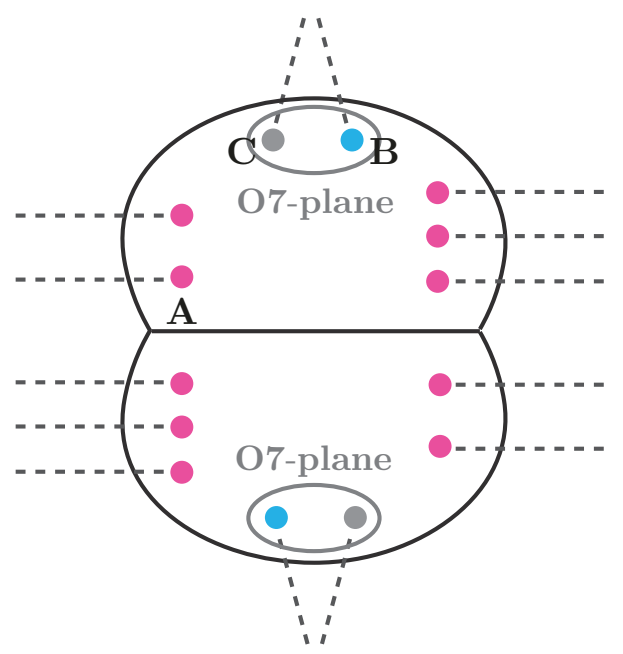

Figure 2. The T-dual description of the type IIA brane setup for $6 \mathrm{~d} \mathcal{N}=(1,0) \operatorname{Sp}(N)$ gauge theory with $2 N+8$ flavors and one tensor multiplet. (For simplicity, the brane description for the $N=1$ case is drawn.) We denote $\mathbf{A}, \mathbf{B}$, and $\mathbf{C}$ for $[1,0] 7$-brane (or D7 brane), [1,-1] 7-brane, and $[1,1]$ 7-brane, respectively. The two $O 7$-planes are replaced by the pair of $\mathbf{B}$ and $\mathbf{C}$. The branch cuts due to 7 -branes are denoted by the dotted lines.

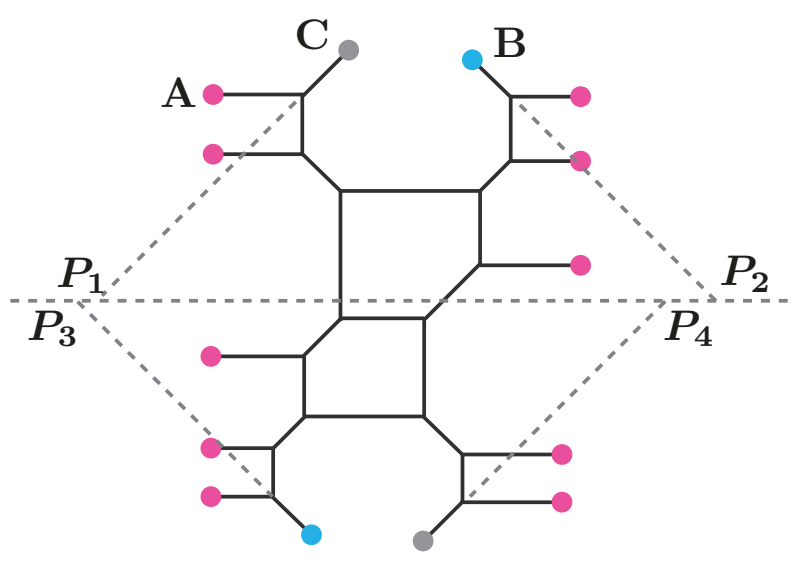

Figure 3. A $(p, q)$-brane setup for $5 \mathrm{~d} \mathrm{SU}(N+2)$ gauge theory with $2 N+8$ flavors. For concreteness, the $N=1$ case is drawn. The horizontal dotted line indicates the center of mass position of $N+2$ color branes. To measure the gauge coupling, one extends the upper and lower $(1,1)$ and $(1,-1)$ 5-branes to the horizontal dotted line, which gives rise to two asymptotic distances between $\mathbf{P}_{\mathbf{1}}$ and $\mathbf{P}_{\mathbf{2}}$, and between $\mathbf{P}_{\mathbf{3}}$ and $\mathbf{P}_{\mathbf{4}}$. The inverse gauge coupling is then given by the average of these two distances.

uppermost and lowermost D5 branes are created. In this diagram, we can explicitly see that there are $N+2$ color D5-branes indicating the $\mathrm{SU}(N+2)$ gauge symmetry. Therefore, we expect that the gauge symmetry is enhanced from $\mathrm{U}(N)$ to $\mathrm{SU}(N+2)$.

In our case, replacing the two $\mathrm{O}^{-}$-planes by two pairs of $[1,1]$ and $[1,-1] 7$-branes has another important aspect. Before this replacement, two $\mathrm{O}^{-}$-planes are located at two places in the compactified circle such that the distance between two $\mathrm{O}^{-}$-planes is the half circumference of the circle. With the replacement, we are changing the picture so that we 


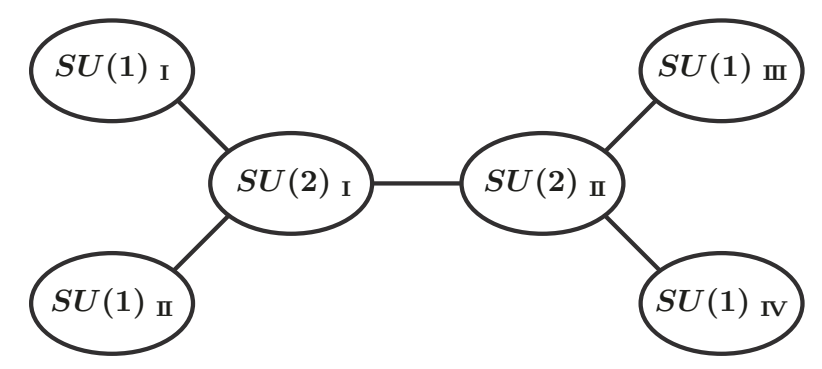

Figure 4. The quiver diagram of the affine $\hat{D}_{5}$ quiver theory.

look at the theory from the purely $5 \mathrm{~d}$ point of view. Hence, the notion of the compactified direction is transmuted into the gauge coupling of the theory. We can see that the radius of the compactified circle is now inherited by the the inverse gauge coupling in the $(p, q) 5$ brane web diagram, which is identified as the average of the asymptotic distances between $(1,1)$ 5-brane and $(1,-1) 5$-brane measured at the center of mass position of $N+2$ color D5-branes by extrapolating them as in figure 3 (up to a numerical factor depending on the convention). Therefore, we expect that the KK mode of the original $6 \mathrm{~d}$ theory compactified on $S^{1}$ is reinterpreted as the instanton contribution in the 5 d theory.

\subsection{Evidence 2: $\hat{D}$-type Dynkin quiver}

It is also possible to justify our conjecture from a different route. The $6 \mathrm{~d}\left(D_{N+4}, D_{N+4}\right)$ minimal conformal matter is realized on a single M5-brane at the orbifold singularity of $\mathbb{C}^{2} / \Gamma_{D_{N+4}}[9]$. An $S^{1}$ compactification of the M5-brane may yield a system of a single D4-brane sitting at the same singularity of $\mathbb{C}^{2} / \Gamma_{D_{N+4}}$ in type IIA string theory. The resulting five-dimensional theory is a $5 \mathrm{~d}$ affine $\hat{D}_{N+4}$ quiver theory. The quiver diagram of the case $N=1$ is depicted in figure 4 . In the quiver diagram, the $N+1$ nodes along the central horizontal line give SU(2) gauge groups, and the 4 nodes on the left-hand and righthand side of the diagram give "SU(1)" gauge groups. Each line which connects two gauge nodes represents a bi-fundamental hypermultiplet. Note that $\mathrm{SO}(2 N+8) \times \mathrm{SO}(2 N+8)$ global symmetry of the six-dimensional theory is broken to a subgroup ${ }^{6}$ due to Wilson line along the $S^{1}$.

Let us comment on the meaning of the "SU(1)" gauge groups. In fact a set of a bifundamental hypermultiplet of $\mathrm{SU}(2) \times \mathrm{SU}(1)$ and an "SU(1)" gauge instanton is equivalent to two fundamental hypermultiplets which are coupled to the $\mathrm{SU}(2)$ gauge group. This has been confirmed in various examples at least at the level of the $5 \mathrm{~d}$ partition functions in $[31,32,40,61]$. Hence, the $5 \mathrm{~d}$ affine $\hat{D}_{N+4}$ quiver theory may be equivalent to a $5 \mathrm{~d}$ $\mathrm{SU}(2)^{N+1}$ linear quiver gauge theory with four fundamental hypermultiplets are coupled to the two $\mathrm{SU}(2)$ gauge group at the left and right ends, respectively. However, in the following discussion, we keep the convention of "SU(1)" gauge group.

We propose that this $5 \mathrm{~d}$ affine $\hat{D}_{N+4}$ quiver theory can be also realized by a web diagram of $(p, q)$ 5-branes in type IIB string theory. The web diagram that we propose

\footnotetext{
${ }^{6}$ However, we will see that there is an enhancement of the global symmetry.
} 


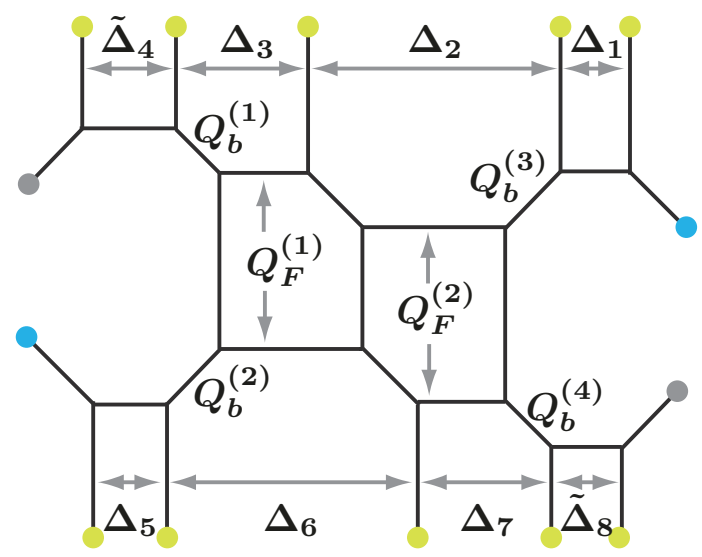

Figure 5. The web diagram which realizes an affine $\hat{D}_{5}$ Dynkin quiver theory. $\mathrm{Q}$ and $\Delta$ assigned to a 5-brane segment in the figure represent $e^{-L}$ where $L$ is the length of the corresponding 5 -brane. This can be also seen as a diagram S-dual to the web diagram for SU(3) gauge theory with 10 flavors.

in the case with $N=1$ is depicted in figure 5. In this web diagram, the two connected closed faces in the middle of the web give $\mathrm{SU}(2) \times \mathrm{SU}(2)$ gauge theory which is coupled by a bi-fundamental hypermultiplet. For the sake of the later computation, let the gauge group from the left loop be $\mathrm{SU}(2)_{\mathrm{I}}$ and the gauge group from the right loop be $\mathrm{SU}(2)_{\mathrm{II}}$. Furthermore, we have four "SU(1)" gauge groups corresponding to four horizontal internal lines with lengths $\Delta_{1}, \tilde{\Delta}_{4}, \Delta_{5}$ and $\tilde{\Delta}_{8}$ in figure 5. Each "SU(1)" is coupled to either of the two $\mathrm{SU}(2)$ gauge groups by a bi-fundamental hypermultiplet. We will denote the four "SU(1)" gauge groups coming from the horizontal lines with lengths $\tilde{\Delta}_{4}, \Delta_{5}, \Delta_{1}$ and $\tilde{\Delta}_{8}$ by $\mathrm{SU}(1)_{\mathrm{I}}, \mathrm{SU}(1)_{\mathrm{II}}, \mathrm{SU}(1)_{\mathrm{III}}$ and $\mathrm{SU}(1)_{\mathrm{IV}}$ respectively. Therefore, we claim that the web diagram in figure 5 exactly yields the affine $\hat{D}_{5}$ Dynkin quiver theory in figure 4 . It is straightforward to write down a web diagram for general $N$. Namely, we put more closed faces along the horizontal directions.

One can give a completely different view by the S-duality rotating the web diagram by 90 degrees. After the S-duality, the web diagram which originally yielded the affine $\hat{D}_{N+4}$ quiver theory becomes the web diagram which realizes the $\mathrm{SU}(N+2)$ gauge theory with $2 N+8$ fundamental hypermultiplets. For the $N=1$ case, one can easily see that the web diagram in figure 5 is nothing but the web diagram in figure 3 after the S-duality. Therefore, we again obtain the $\mathrm{SU}(N+2)$ gauge theory with $2 N+8$ flavors from the $S^{1}$ compactification of the six-dimensional theory on a single M5-brane at the origin of $\mathbb{C}^{2} / \Gamma_{D_{N+4}}$.

From this point of view of the affine $\hat{D}_{N+4}$ Dynkin quiver theory, one can further give a quantitative support for our conjecture. For simplicity, let us focus on the case with $N=1$. Since the $S^{1}$ compactification gives the $5 \mathrm{~d}$ affine $\hat{D}_{5}$ quiver theory, an appropriate product of the instanton fugacities of the quiver theory is expected to be equal to the fugacity for the KK U(1) symmetry. The exponents of the instanton fugacity of a gauge node are the comarks. The exponent for instanton fugacity associated to the extended node is 1 , for 

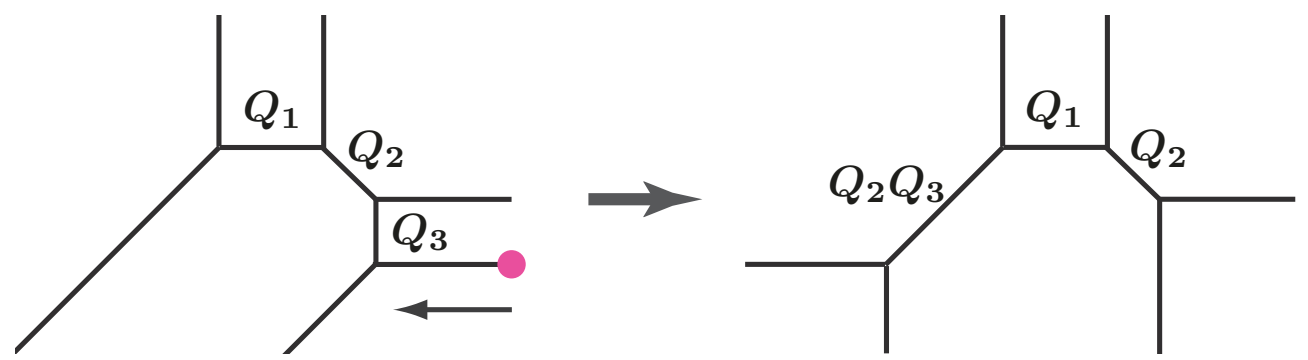

Figure 6. Left: the web diagram which gives an "SU(1)" gauge theory. Right: another equivalent web diagram which is given by moving the 7-brane in the left figure. This figure makes it easy to identify the instanton fugacity for the "SU(1)".

instance. More specifically, the following relation should hold for the affine $\hat{D}_{5}$ quiver,

$$
q^{\mathrm{SU}(1)_{\mathrm{I}}} q^{\mathrm{SU}(1)_{\mathrm{II}}}\left(q^{\mathrm{SU}(2)_{\mathrm{I}}}\right)^{2}\left(q^{\mathrm{SU}(2)_{\mathrm{II}}}\right)^{2} q^{\mathrm{SU}(1)_{\mathrm{III}}} q^{\mathrm{SU}(1)_{\mathrm{IV}}}=\Delta,
$$

where $q^{G}$ is the instanton fugacity for a gauge group $G$, and $\Delta$ is the fugacity for the KK $\mathrm{U}(1)$ symmetry. In the following, we discuss that the relation (2.1) is indeed satisfied from the web diagram in figure 5 .

The instanton fugacity of each gauge group can be easily inferred from the web diagram. Namely it is given by an exponential of the average of the sum of the asymptotic distances between two NS5-branes extending in the upper and the lower part of diagram (see for example [62]). Hence, the instanton fugacities for the two $\mathrm{SU}(2)$ gauge groups are

$$
q^{\mathrm{SU}(2)_{\mathrm{I}}}=\left(\Delta_{3} \Delta_{6}\right)^{\frac{1}{2}}, \quad q^{\mathrm{SU}(2)_{\mathrm{II}}}=\left(\Delta_{2} \Delta_{7}\right)^{\frac{1}{2}} .
$$

The instanton fugacities of the "SU(1)" gauge groups can be understood in a similar way. Let us consider the diagram in figure 6. The identification of the instanton fugacity of the "SU(1)" gauge group is less obvious in the left diagram in figure 6. However, one can move the 7-brane, and after the Hanany-Witten transition one arrives at the diagram on the right hand side of figure 6 which gives an equivalent theory. From the diagram on the right, it is then easy to identify the asymptotic distances between two NS5 branes: the upper and lower distances are $Q_{1}$ and $Q_{1} Q_{2}^{2} Q_{3}$, respectively. The instanton fugacity is thus given by

$$
q^{\mathrm{SU}(1)}=Q_{1} Q_{2} Q_{3}^{\frac{1}{2}}
$$

One can apply the rule (2.3) to the instanton fugacity of each of the four "SU(1)" gauge groups in figure 5 , and the result is

$$
\begin{aligned}
q^{\mathrm{SU}(1)_{\mathrm{I}}} & =\tilde{\Delta}_{4} Q_{b}^{(1)} Q_{F}^{(1) \frac{1}{2}}, & q^{\mathrm{SU}(1)_{\mathrm{II}}} & =\Delta_{5} Q_{b}^{(2)} Q_{F}^{(1) \frac{1}{2}}, \\
q^{\mathrm{SU}(1)_{\mathrm{III}}} & =\Delta_{1} Q_{b}^{(3)} Q_{F}^{(2) \frac{1}{2}}, & q^{\mathrm{SU}(1)_{\mathrm{IV}}} & =\tilde{\Delta}_{8} Q_{b}^{(4)} Q_{F}^{(2) \frac{1}{2}}
\end{aligned}
$$

To relate them with the $\mathrm{KK} \mathrm{U}(1)$ fugacity $\Delta$, one needs to move to the Tao diagram from figure 5 by using a sequence of Hanany-Witten transition and moving out all the 7-branes to infinity as discussed in [50]. For example, from figure 5, one can perform a flop transition associated with $Q_{b}^{(2)}$ as well as $Q_{b}^{(3)}$, and then do successive Hanany-Witten 


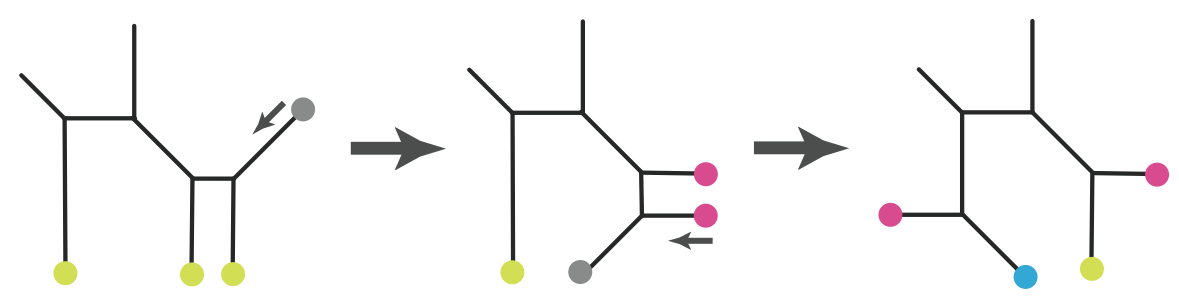

Figure 7. An example of successive Hanany-Witten transition applied on figure 5 which makes a Tao configuration.

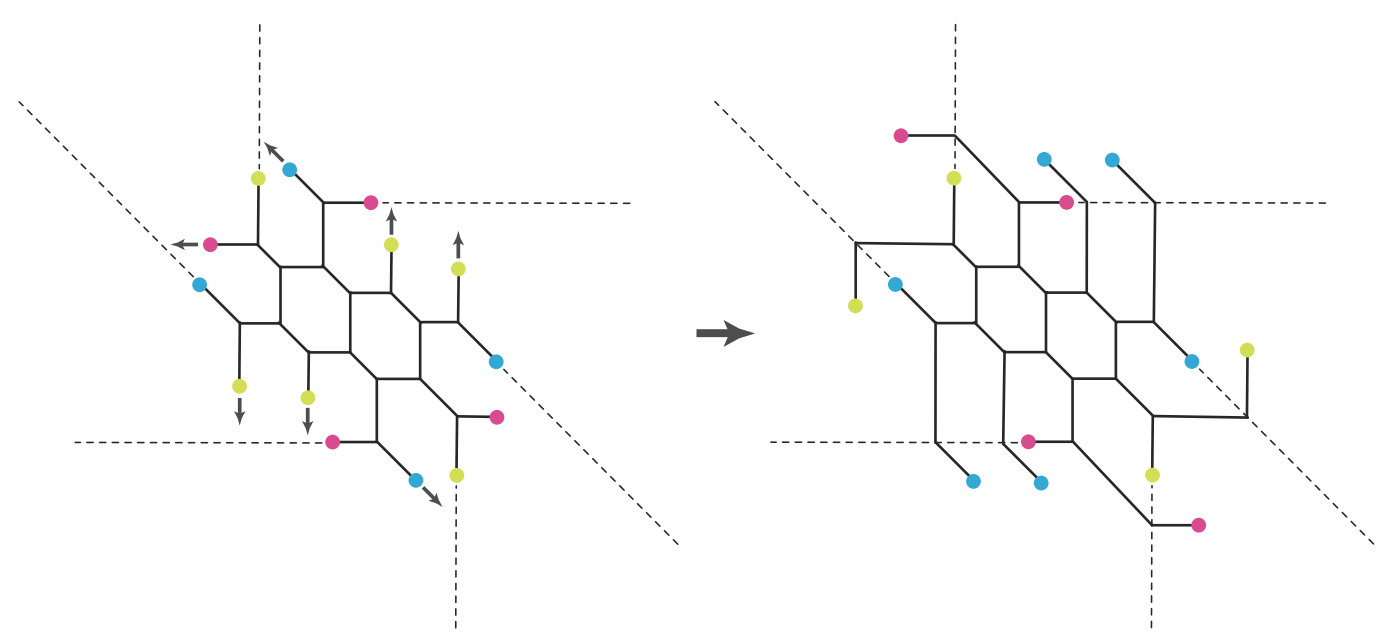

Figure 8. The 7-brane motions leading to Tao web diagram for the affine $\hat{D}_{5}$ Dynkin diagram.

transition on the right bottom and on the left top 7-branes involving [1,1] 7-brane (gray dot) and three $[0,1]$ 7-branes (dark yellow dots) as in figure 7 . After choosing proper branch cuts as in figure 8 , one takes all the 7 -branes to infinity to obtain a Tao diagram depicted in figure 9 . In this web diagram description, the fugacity $\Delta$ is given by the period of the spiral diagram [50]. The period of the Tao diagram in figure 9 is simply given by

$$
\Delta=\Delta_{1} \Delta_{2} \Delta_{3} \Delta_{4} \Delta_{5} \Delta_{6} \Delta_{7} \Delta_{8}
$$

From figure 9 , it is also possible to identify $\Delta_{4}, \Delta_{8}$ by the parameters in figure 5 , and the result is

$$
\Delta_{4}=\tilde{\Delta}_{4} Q_{b}^{(1)} Q_{b}^{(2)} Q_{F}^{(1)}, \quad \Delta_{8}=\tilde{\Delta}_{8} Q_{b}^{(3)} Q_{b}^{(4)} Q_{F}^{(2)} .
$$

Using (2.2), (2.4) and (2.6), one can rewrite the left hand side of (2.1) as

$$
q^{\mathrm{SU}(1)_{\mathrm{I}}} q^{\mathrm{SU}(1)_{\mathrm{II}}}\left(q^{\mathrm{SU}(2)_{\mathrm{I}}}\right)^{2}\left(q^{\mathrm{SU}(2)_{\mathrm{II}}}\right)^{2} q^{\mathrm{SU}(1)_{\mathrm{III}}} q^{\mathrm{SU}(1)_{\mathrm{IV}}}=\Delta_{1} \Delta_{2} \Delta_{3} \Delta_{4} \Delta_{5} \Delta_{6} \Delta_{7} \Delta_{8},
$$

which is exactly equal to $\Delta$ due to (2.5). Hence, the relation (2.1) indeed holds for the affine $\hat{D}_{5}$ Dynkin web diagram in figure 5 . The same argument in fact holds for the $\mathrm{SU}(3)$ gauge theory with 10 flavors. The generalization of the computation to general $N$ is also straightforward. 


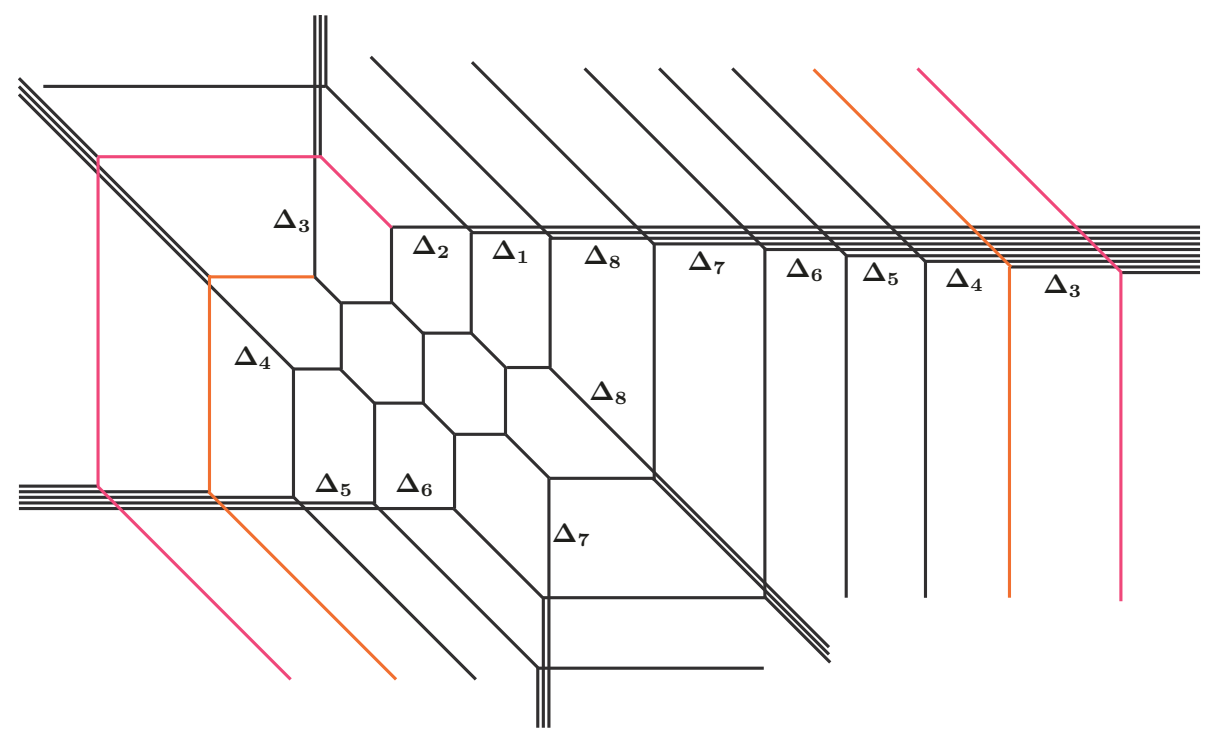

Figure 9. The Tao web diagram corresponding to the affine $\hat{D}_{5}$ Dynkin diagram in figure 5 .

\section{Global symmetry of SU( $n)$ gauge theories}

The classification of the UV complete $5 \mathrm{~d} \mathrm{SU}(n)$ theories ${ }^{7}$ with $n>2$ and $N_{f}$ fundamental hypermultiplets has been done in [63] which states that $5 \mathrm{~d}$ UV fixed point exists for $N_{f} \leq$ $2 n$. On the other hand, it was conjectured in [40] that the 5d UV fixed point exists for

$$
N_{f}<2 n+4, \quad \text { and } \quad N_{f} \leq 2 n+4-2|\kappa|,
$$

where $\kappa$ is the Chern-Simons (CS) level. For $n=3$, the $(p, q) 5$-brane web for $N_{f} \leq 6$ is straightforward. The web diagrams for $N_{f}=7$ with $\kappa=\frac{1}{2}, \frac{3}{2}$ and $N_{f}=8$ with $\kappa=0$ are given in [40]. Here we strengthen this conjecture by finding the additional diagrams for $N_{f}=9$ with $\kappa=\frac{1}{2}$ and $N_{f}=8$ with $\kappa=1$ in figure 10 .

The insight of [50] is that one can extend the class of the web diagrams by including Tao diagrams where 5-branes has an infinitely expanding spiral configuration, and the corresponding $5 \mathrm{~d}$ theories are expected to be UV complete and have a $6 \mathrm{~d}$ fixed point. From this point of view, our conjecture is that the UV fixed point of the $5 \mathrm{~d} \mathrm{SU}(n)$ gauge theory which satisfies the bound $N_{f}=2 n+4$ exists due to the existence of the corresponding Tao diagram, ${ }^{8}$ and this UV SCFT is precisely the $6 \mathrm{~d}\left(D_{n+2}, D_{n+2}\right)$ minimal conformal matter. This is essentially a generalization of the claim for the known $n=2$ case.

In the $n=2$ case, the global symmetry is enhanced either at the $5 \mathrm{~d}$ fixed point or the $6 \mathrm{~d}$ fixed point depending on the UV completion. In this section, we scrutinize the global symmetry enhancement for $5 \mathrm{~d} \mathrm{SU}(n)$ gauge theory with $n>2$ and $2 n \leq N_{f} \leq 2 n+4$ flavors together with the CS level $\kappa$ subject to (3.1) We also list up the non-abelian part of the global symmetries of all the possible $\mathrm{SU}(3)$ gauge theories with flavors and various

\footnotetext{
${ }^{7}$ Throughout this section, instead of $\mathrm{SU}(N+2)$ gauge theories used in the previous section, we use $\mathrm{SU}(n)$ for denoting the $5 \mathrm{~d}$ gauge group for simplicity. To relate to section 2 , one can take $n=N+2$.

${ }^{8}$ For example, the Tao diagram of $n=3$ is given by figure 9 .
} 

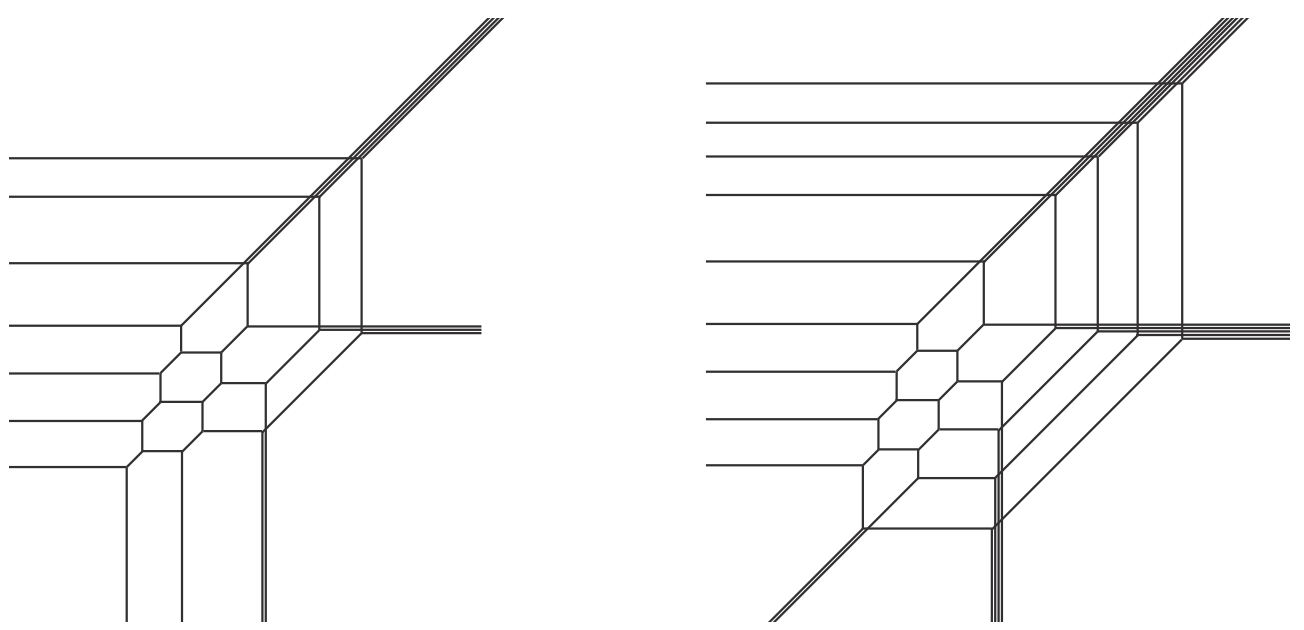

Figure 10. A web diagram of SU(3) theory with $N_{f}=8$ flavors with CS level 1 (left) and with $N_{f}=9$ flavors with CS level $\frac{1}{2}$ (right). They are obtained by taking mass decoupling limit from Tao diagram.

CS levels which have the UV fixed point. We employ the 7-brane technology developed by $[27,64,65]$ to characterize the global symmetry structure for various flavors.

Given two 7-branes of whose configuration is denoted by $\mathbf{X}_{\boldsymbol{z}_{1}}$ and $\mathbf{X}_{\boldsymbol{z}_{2}}$ with two charge vectors $\boldsymbol{z}_{i}=\left[p_{i}, q_{i}\right]$, the changes in the charge of one 7-brane due to crossing the branch cut of the adjoining 7-brane are given by

$$
\mathbf{X}_{\boldsymbol{z}_{1}} \mathbf{X}_{\boldsymbol{z}_{2}}=\mathbf{X}_{\boldsymbol{z}_{2}} \mathbf{X}_{\boldsymbol{z}_{1}+\left(\boldsymbol{z}_{1} \wedge \boldsymbol{z}_{2}\right) \boldsymbol{z}_{2}}=\mathbf{X}_{\boldsymbol{z}_{2}+\left(\boldsymbol{z}_{1} \wedge \boldsymbol{z}_{2}\right) \boldsymbol{z}_{1}} \mathbf{X}_{\boldsymbol{z}_{1}}
$$

where $\boldsymbol{z}_{1} \wedge \boldsymbol{z}_{2}=p_{1} q_{2}-p_{2} q_{1}$. As we will see below, a 5-brane web can be recast into 7 -brane configuration with 5 -brane loop probing it. The 7-brane configuration carries the enhanced global symmetry, and one can identify the symmetry by extracting the 7-brane configurations which collapse into $\mathbf{A}_{m}, \mathbf{D}_{m}, \mathbf{E}_{m}$ Kodaira singularities of which realize $A_{m}$, $D_{m}, E_{m}$ algebras. Rearranging 7 -branes with the above reordering rule enables us to extract such basic 7-brane configurations. If we use a short hand notation for frequently appearing 7-branes:

$$
\mathbf{A}=[1,0], \quad \mathbf{B}=[1,-1], \quad \mathbf{C}=[1,1], \quad \mathbf{N}=[0,1]
$$

the (enhanced) global symmetry can be read off from the Kodaira classification

$$
\begin{aligned}
\mathbf{A}_{m}: & \mathbf{A}^{m+1}, \\
\mathbf{D}_{m \geq 4}: & \mathbf{A}^{m} \mathbf{B C}, \\
\mathbf{E}_{m \geq 6}: & \mathbf{A}^{m-1} \mathbf{B C C},
\end{aligned}
$$

up to $\mathrm{SL}(2, \mathbb{Z})$ transformation.

$\mathrm{SU}(\boldsymbol{n})_{0}, \boldsymbol{N}_{\boldsymbol{f}}=\mathbf{2 n}+\mathbf{4}$ theory. Let us first start from the $5 \mathrm{~d} \mathrm{SU}(n)$ gauge theory with $N_{f}=2 n+4$ and the vanishing CS level. An example of the web configuration for the $\mathrm{SU}(3)$ 


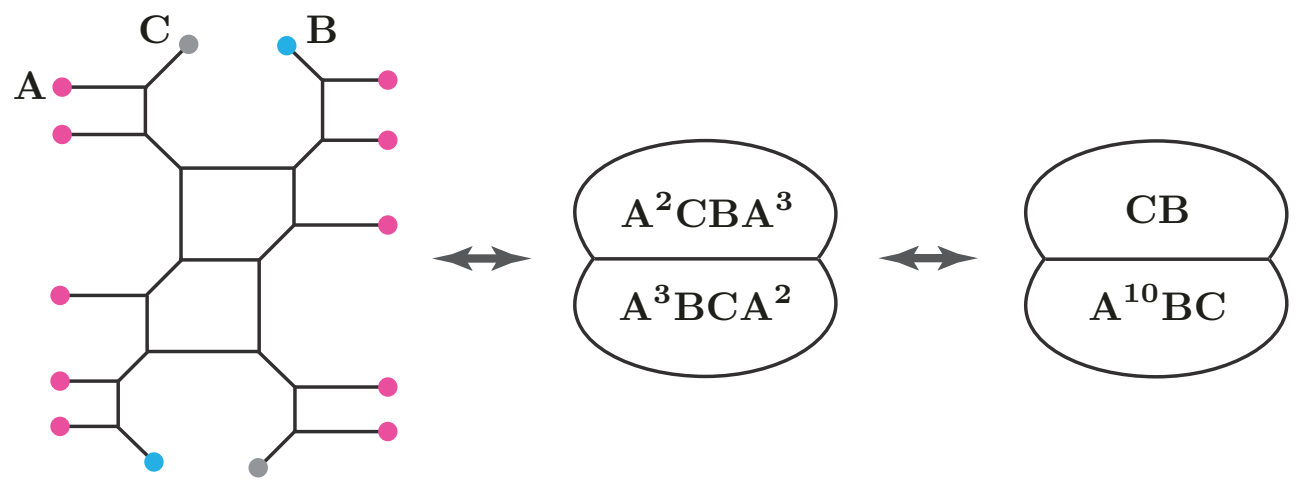

Figure 11. A web diagram of SU(3) theory with $N_{f}=10$ flavors in which 5-branes attached to 7-branes labeled by their charge vectors $\mathbf{A}, \mathbf{B}, \mathbf{C}, \mathbf{N}$ is given on the left. The middle and right diagrams are the corresponding 7-brane configuration leading to the $\mathbf{D}_{10}=\mathbf{A}^{10} \mathbf{B C}$ corresponding to $\mathrm{SO}(20)$ global symmetry.

theory with $N_{f}=10$ flavors is given in figure 11. In order to read off the global symmetry, we pull in all the 7-branes either inside the uppermost 5-brane loop or the lowermost the 5-brane loop. We will describe the 7-brane configuration as "(lower| upper)". The configuration of 7 -branes for the $\mathrm{SU}(n)$ gauge theories with $N_{f}=2 n+4$ is then, by listing 7-branes counterclockwise from the left $n$ D7-branes, given by

$$
\left(\mathbf{A}^{n} \mathbf{B C A}^{2} \mid \mathbf{A}^{n} \mathbf{B C A}^{2}\right) .
$$

Recall that the position of a flavor brane leads to the mass parameter of the hypermultiplet and by mass deformation its position can be relocated through the different Coulomb moduli. D7-branes can cross D5-branes without affecting them, and this means that the configuration $\mathbf{A}$ is not confined within a 5-brane loop. Together with 7-brane monodromies, e.g.,

$$
\mathbf{A B}=\mathbf{B N}, \quad \mathbf{N C}=\mathbf{C A}, \quad \Rightarrow \quad \mathbf{A B C}=\mathbf{B C A},
$$

one easily finds that the 7-brane configuration (3.5) becomes

$$
\left(\mathbf{A}^{2 n+4} \mathbf{B C} \mid \mathbf{B C}\right) \text {. }
$$

Hence, this gives an $\mathrm{SO}(4 n+8)$ global symmetry which is enhanced from $\mathrm{U}(2 n+4)$ symmetry of $2 n+4$ flavors. It should be emphasized that Tao diagram contains spiral rotations associated with KK modes whose period is the instanton factor. The enhanced global symmetry for $N_{f}=2 n+4$ flavors is then

$$
\mathrm{SO}(4 n+8) \times \mathrm{U}(1)_{\mathrm{I}} \supset \mathrm{U}(2 n+4) \times \mathrm{U}(1)_{\mathrm{I}},
$$

where $\mathrm{U}(1)_{\mathrm{I}}$ is symmetry of instanton particle coming from $S^{1}$ compactification.

$\mathbf{S U}(\boldsymbol{n})_{\frac{1}{2}}, \boldsymbol{N}_{\boldsymbol{f}}=\mathbf{2} \boldsymbol{n}+\mathbf{3}$ theory. We then decouple one flavor and consider an $\mathrm{SU}(n)$ gauge theory with $N_{f}=2 n+3$ and the CS level $\kappa= \pm \frac{1}{2}$. An example of the 5-brane web diagram for $\mathrm{SU}(3)$ gauge theory with $N_{f}=9$ flavors is given in figure 12 which can be 


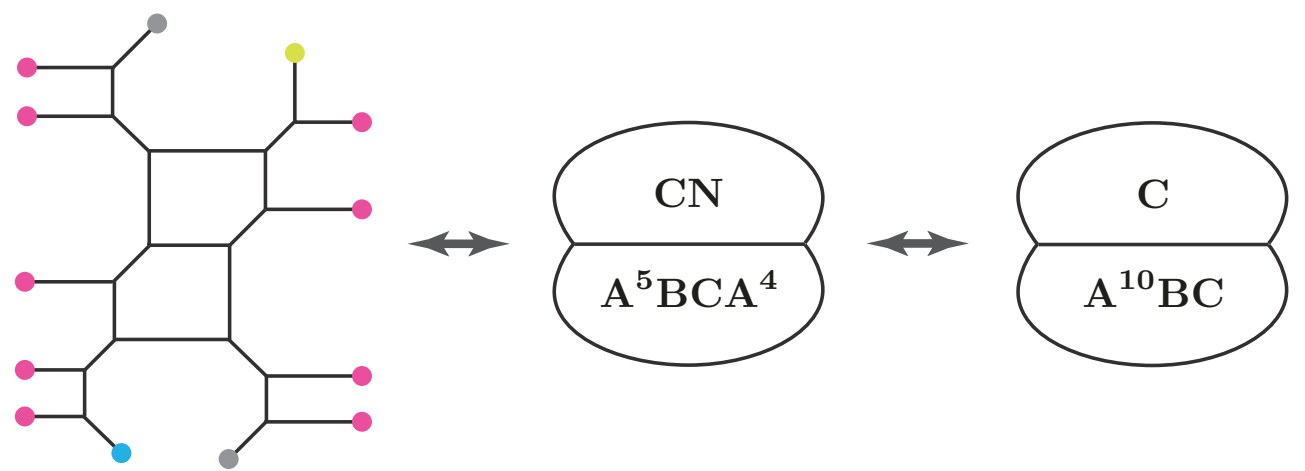

Figure 12. A web diagram of SU(3) theory with $N_{f}=9$ flavors in which 5-branes attached to 7-branes labeled by their charge vectors $\mathbf{A}, \mathbf{B}, \mathbf{C}, \mathbf{D}$, and 7-brane configuration leading to the $\mathbf{D}_{10}=\mathbf{A}^{10} \mathbf{B C}$ corresponding to $\mathrm{SO}(20)$ global symmetry.

understood as the mass decoupling limit where the position of one of flavor D5-branes is taken to infinity. As can be read off from figure 12, it has the CS level $\kappa=-\frac{1}{2}$. The web diagram is now no longer a Tao diagram and it is a usual web diagram of $(p, q)$ 5-branes. Hence, we expect that its UV completion is a 5d SCFT and it has an enhancement of the global symmetry at the fixed point. The global symmetry of the $\mathrm{SU}(n)$ gauge theory with $N_{f}=2 n+3$ and $\kappa=-\frac{1}{2}$ is again dictated in the 7-brane monodromy

$$
\left(\mathbf{A}^{n} \mathbf{B C A} \mathbf{A}^{2} \mid \mathbf{A}^{n-1} \mathbf{N C A}{ }^{2}\right)=\left(\mathbf{A}^{n+2} \mathbf{B C} \mathbf{A}^{n+1} \mid \mathbf{N C}\right)=\left(\mathbf{A}^{2 n+3} \mathbf{B C} \mid \mathbf{C A}\right)=\left(\mathbf{A}^{2 n+4} \mathbf{B C} \mid \mathbf{C}\right),
$$

where we used (3.6), $\mathbf{N C}=\mathbf{C A}$, which provides an additional A 7-brane thus yielding enhanced $\mathrm{SO}(2 n+4)$ symmetry

$$
\mathrm{SO}(2 n+4) \supset \mathrm{U}(2 n+3) \times \mathrm{U}(1) .
$$

The $\kappa=\frac{1}{2}$ case also gives rise to the same enhanced global symmetry. As the CS level for $5 \mathrm{~d}$ theories is subjected to (3.1), the allowed CS levels for $N_{f}=2 n+3$ flavors are only $\kappa= \pm \frac{1}{2}$. For less flavors, more CS levels are allowed and the corresponding global symmetry may have different enhanced global symmetry depending on the CS level.

$\mathrm{SU}(n)_{\kappa}, \mathbf{2 n} \leq \boldsymbol{N}_{\boldsymbol{f}} \leq \mathbf{2 n + 2}$ theory. Using 7-brane monodromies, it is straightforward to generalize to $\mathrm{SU}(n)$ gauge theories with $2 n \leq N_{f} \leq 2 n+2$ and the CS level which satisfies (3.1) by decoupling the flavors one by one. The resulting theories are supposed to have the 5d UV fixed point. It is convenient to label the corresponding global symmetries by the CS level $|\kappa|$, like $G_{|\kappa|}$, and the enhanced global symmetries $G_{|\kappa|}$ of the theories are summarized in table 1.

We also included the cases with $N_{f}=2 n+3,2 n+4$ for completeness. In the table 1 , we only wrote the non-abelian part of the enhanced global symmetries. The abelian part can be easily reproduced since the total number of the rank of the global symmetry should be $N_{f}+1$, which corresponds to the number of the parameters of the theory. The theories exhaust all the possibilities with $N_{f} \geq 2 n$ which satisfy (3.1), and all the theories show the enhancement of the global symmetries. 


\begin{tabular}{|c|ll|}
\hline$N_{f}$ & $G_{|\kappa|}$ \\
\hline $2 n+4$ & $\mathrm{SO}(4 n+8)_{0}$ & \\
$2 n+3$ & $\mathrm{SO}(4 n+8)_{\frac{1}{2}}$ & \\
$2 n+2$ & $\mathrm{SU}(2 n+4)_{0}, \quad[\mathrm{SO}(4 n+4) \times \mathrm{SU}(2)]_{1}$ & \\
$2 n+1$ & {$[\mathrm{SU}(2 n+2) \times \mathrm{SU}(2)]_{\frac{1}{2}}, \quad \mathrm{SO}(4 n+2)_{\frac{3}{2}}$} & \\
$2 n$ & {$[\mathrm{SU}(2 n) \times \mathrm{SU}(2) \times \mathrm{SU}(2)]_{0}, \quad \mathrm{SU}(2 n+1)_{1}, \quad \mathrm{SO}(4 n)_{2}$} \\
\hline
\end{tabular}

Table 1. The non-abelian part of the enhanced global symmetries $G_{|\kappa|}$ of $5 \mathrm{~d} \mathrm{SU}(n)_{\kappa}$ gauge theories with $N_{f} \geq 2 n$ at UV fixed point.

\begin{tabular}{|c|c|}
\hline$N_{f}$ & $G_{|\kappa|}(\kappa$ is the Chern-Simons level $)$ \\
\hline 10 & $\mathrm{SO}(20)_{0}$ \\
\hline 9 & $\mathrm{SO}(20)_{\frac{1}{2}}$ \\
\hline 8 & $\mathrm{SU}(10)_{0}, \quad[\mathrm{SO}(16) \times \mathrm{SU}(2)]_{1}$ \\
\hline 7 & {$[\mathrm{SU}(8) \times \mathrm{SU}(2)]_{\frac{1}{2}}, \quad \mathrm{SO}(14)_{\frac{3}{2}}$} \\
\hline 6 & {$[\mathrm{SU}(6) \times \mathrm{SU}(2) \times \mathrm{SU}(2)]_{0}, \quad \mathrm{SU}(7)_{1}, \quad \mathrm{SO}(12)_{2}$} \\
\hline 5 & {$[\mathrm{SU}(5) \times \mathrm{SU}(2)]_{\frac{1}{2}}, \quad \mathrm{SU}(6)_{\frac{3}{2}}, \quad \mathrm{SO}(10)_{\frac{5}{2}}$} \\
\hline 4 & $\mathrm{SU}(4)_{0}, \quad[\mathrm{SU}(4) \times \mathrm{SU}(2)]_{1}, \quad \mathrm{SU}(5)_{2}, \quad \mathrm{SO}(8)_{3}$ \\
\hline 3 & $\mathrm{SU}(3)_{\frac{1}{2}}, \quad[\mathrm{SU}(3) \times \mathrm{SU}(2)]_{\frac{3}{2}}, \quad \mathrm{SU}(4)_{\frac{5}{2}}, \quad \mathrm{SO}(6)_{\frac{7}{2}}$ \\
\hline 2 & $\mathrm{SU}(2)_{0}, \quad \mathrm{SU}(2)_{1}, \quad[\mathrm{SU}(2) \times \mathrm{SU}(2)]_{2}, \quad \mathrm{SU}(3)_{3}, \quad \mathrm{SO}(4)_{4}$ \\
\hline 1 & $\mathrm{SU}(2)_{\frac{5}{2}}, \quad \mathrm{SU}(2)_{\frac{7}{2}}$ \\
\hline 0 & $\mathrm{SU}(2)_{3}$ \\
\hline
\end{tabular}

Table 2. The non-abelian part of the global symmetries of all the $5 \mathrm{~d}$ SU(3) gauge theories which have the UV completion. The theories that do not have non-abelian global symmetry are omitted.

It is straightforward to check the global symmetry of $\mathrm{SU}(n)_{\kappa}$ theory for lower flavors, and there are various global symmetries depending on the CS level $\kappa$ at a given number flavor. It is worth of noting that for $N_{f}=0,1$ cases, however, not all CS level lead to non-abelian global symmetry; it appears only for the following CS levels (denoted in the subscript):

$$
N_{f}=0: \mathrm{SU}(2)_{n}, \quad N_{f}=1: \mathrm{SU}(2)_{n-\frac{1}{2}}, \mathrm{SU}(2)_{n+\frac{1}{2}}
$$

$\mathrm{SU}(3)_{\kappa}, N_{f}$ theory with $\boldsymbol{N}_{\boldsymbol{f}} \leq \mathbf{1 0}$ flavors. As a concrete example of the enhanced global symmetries for a given $\mathrm{SU}(n)$ gauge theory, we pick $n=3$ and list all the global symmetries of $5 \mathrm{~d} \mathrm{SU}(3)$ gauge theory of all the allowed flavors and the CS level $\kappa$ which has the UV completion in table 2 .

We wrote down only the non-abelian part in table 2. There is again an abelian part such that the total rank of the global symmetry is $N_{f}+1$. Bergman et al. have found a considerable part of the list of the enhanced global symmetry [34, 40]. Here we enclose all for the completeness. Our results for $N_{f}=10,9$ and a few higher Chern-Simons level cases seem new. 


\section{Acknowledgments}

We thank Amihay Hanany, Seok Kim, Ashoke Sen and David Tong for useful discussions. The work of H.H. is supported by the grant FPA2012-32828 from the MINECO, the REA grant agreement PCIG10-GA-2011-304023 from the People Programme of FP7 (Marie Curie Action), the ERC Advanced Grant SPLE under contract ERC-2012-ADG20120216-320421 and the grant SEV-2012-0249 of the "Centro de Excelencia Severo Ochoa" Programme. The work of K.L. is supported in part by the National Research Foundation of Korea (NRF) Grants No. 2006-0093850. The work of M.T. is supported by the RIKEN iTHES project.

Open Access. This article is distributed under the terms of the Creative Commons Attribution License (CC-BY 4.0), which permits any use, distribution and reproduction in any medium, provided the original author(s) and source are credited.

\section{References}

[1] B. Haghighat, A. Iqbal, C. Kozçaz, G. Lockhart and C. Vafa, M-strings, Commun. Math. Phys. 334 (2015) 779 [arXiv:1305.6322] [InSPIRE].

[2] B. Haghighat, C. Kozçaz, G. Lockhart and C. Vafa, Orbifolds of M-strings, Phys. Rev. D 89 (2014) 046003 [arXiv:1310.1185] [InSPIRE].

[3] B. Haghighat, G. Lockhart and C. Vafa, Fusing E-strings to heterotic strings: $E+E \rightarrow H$, Phys. Rev. D 90 (2014) 126012 [arXiv:1406.0850] [INSPIRE].

[4] J. Kim, S. Kim, K. Lee, J. Park and C. Vafa, Elliptic genus of E-strings, arXiv:1411.2324 [INSPIRE].

[5] B. Haghighat, A. Klemm, G. Lockhart and C. Vafa, Strings of minimal 6d SCFTs, Fortschr. Phys. 63 (2015) 294 [arXiv:1412.3152] [INSPIRE].

[6] A. Gadde et al., 6d string chains, arXiv:1504.04614 [INSPIRE].

[7] J.J. Heckman, D.R. Morrison and C. Vafa, On the classification of 6D SCFTs and generalized ADE orbifolds, JHEP 05 (2014) 028 [Erratum ibid. 06 (2015) 017] [arXiv: 1312.5746] [INSPIRE].

[8] D. Gaiotto and A. Tomasiello, Holography for $(1,0)$ theories in six dimensions, JHEP 12 (2014) 003 [arXiv: 1404.0711] [INSPIRE].

[9] M. Del Zotto, J.J. Heckman, A. Tomasiello and C. Vafa, 6d conformal matter, JHEP 02 (2015) 054 [arXiv: 1407.6359] [INSPIRE].

[10] J.J. Heckman, More on the matter of 6D SCFTs, Phys. Lett. B 747 (2015) 73 [arXiv: 1408.0006] [INSPIRE].

[11] J.J. Heckman, D.R. Morrison, T. Rudelius and C. Vafa, Atomic classification of 6D SCFTs, Fortschr. Phys. 63 (2015) 468 [arXiv: 1502.05405] [InSPIRE].

[12] L. Bhardwaj, Classification of $6 d \mathcal{N}=(1,0)$ gauge theories, arXiv:1502.06594 [INSPIRE].

[13] K. Ohmori, H. Shimizu, Y. Tachikawa and K. Yonekura, $6 d \mathcal{N}=(1,0)$ theories on $T^{2}$ and class S theories. Part I, JHEP 07 (2015) 014 [arXiv: 1503.06217] [INSPIRE]. 
[14] M. Del Zotto, C. Vafa and D. Xie, Geometric engineering, mirror symmetry and $6 d_{(1,0)} \rightarrow 4 d_{(\mathcal{N}=2)}$, arXiv: 1504.08348 [INSPIRE].

[15] K. Ohmori, H. Shimizu and Y. Tachikawa, Anomaly polynomial of E-string theories, JHEP 08 (2014) 002 [arXiv: 1404.3887] [INSPIRE].

[16] K. Ohmori, H. Shimizu, Y. Tachikawa and K. Yonekura, Anomaly polynomial of general 6d SCFTs, Prog. Theor. Exp. Phys. 2014 (2014) 103B07 [arXiv: 1408.5572] [InSPIRE].

[17] K. Intriligator, 6d, $\mathcal{N}=(1,0)$ Coulomb branch anomaly matching, JHEP 10 (2014) 162 [arXiv: 1408.6745] [INSPIRE].

[18] J.J. Heckman, D.R. Morrison, T. Rudelius and C. Vafa, Geometry of 6D RG flows, arXiv: 1505.00009 [INSPIRE].

[19] M. Del Zotto, J.J. Heckman, D.R. Morrison and D.S. Park, 6D SCFTs and gravity, JHEP 06 (2015) 158 [arXiv: 1412.6526] [INSPIRE].

[20] M. Del Zotto, J.J. Heckman, D.S. Park and T. Rudelius, On the defect group of a 6D SCFT, arXiv: 1503.04806 [INSPIRE].

[21] R.-X. Miao, Universal terms of entanglement entropy for 6d CFTs, arXiv:1503.05538 [INSPIRE].

[22] O.J. Ganor and A. Hanany, Small E $E_{8}$ instantons and tensionless noncritical strings, Nucl. Phys. B 474 (1996) 122 [hep-th/9602120] [INSPIRE].

[23] N. Seiberg and E. Witten, Comments on string dynamics in six-dimensions, Nucl. Phys. B 471 (1996) 121 [hep-th/9603003] [INSPIRE].

[24] N. Seiberg, Five-dimensional SUSY field theories, nontrivial fixed points and string dynamics, Phys. Lett. B 388 (1996) 753 [hep-th/9608111] [INSPIRE].

[25] M.R. Douglas, S.H. Katz and C. Vafa, Small instantons, Del Pezzo surfaces and type-I-prime theory, Nucl. Phys. B 497 (1997) 155 [hep-th/9609071] [INSPIRE].

[26] D.R. Morrison and N. Seiberg, Extremal transitions and five-dimensional supersymmetric field theories, Nucl. Phys. B 483 (1997) 229 [hep-th/9609070] [INSPIRE].

[27] O. DeWolfe, A. Hanany, A. Iqbal and E. Katz, Five-branes, seven-branes and five-dimensional $E_{n}$ field theories, JHEP 03 (1999) 006 [hep-th/9902179] [INSPIRE].

[28] H.-C. Kim, S.-S. Kim and K. Lee, 5-dim superconformal index with enhanced $E_{n}$ global symmetry, JHEP 10 (2012) 142 [arXiv:1206.6781] [INSPIRE].

[29] A. Iqbal and C. Vafa, BPS degeneracies and superconformal index in diverse dimensions, Phys. Rev. D 90 (2014) 105031 [arXiv:1210.3605] [inSPIRE].

[30] O. Bergman, D. Rodríguez-Gómez and G. Zafrir, Discrete $\theta$ and the $5 d$ superconformal index, JHEP 01 (2014) 079 [arXiv:1310.2150] [INSPIRE].

[31] L. Bao, V. Mitev, E. Pomoni, M. Taki and F. Yagi, Non-Lagrangian theories from brane junctions, JHEP 01 (2014) 175 [arXiv:1310.3841] [INSPIRE].

[32] H. Hayashi, H.-C. Kim and T. Nishinaka, Topological strings and $5 d T_{N}$ partition functions, JHEP 06 (2014) 014 [arXiv: 1310.3854] [INSPIRE].

[33] M. Taki, Notes on enhancement of flavor symmetry and 5d superconformal index, arXiv: 1310.7509 [INSPIRE]. 
[34] O. Bergman, D. Rodríguez-Gómez and G. Zafrir, 5-brane webs, symmetry enhancement and duality in 5d supersymmetric gauge theory, JHEP 03 (2014) 112 [arXiv:1311.4199] [INSPIRE].

[35] M. Taki, Seiberg duality, 5d SCFTs and Nekrasov partition functions, arXiv:1401.7200 [INSPIRE].

[36] C. Hwang, J. Kim, S. Kim and J. Park, General instanton counting and 5d SCFT, JHEP 07 (2015) 063 [arXiv: 1406.6793] [INSPIRE].

[37] G. Zafrir, Duality and enhancement of symmetry in 5d gauge theories, JHEP 12 (2014) 116 [arXiv: 1408.4040] [INSPIRE].

[38] H. Hayashi and G. Zoccarato, Exact partition functions of Higgsed $5 d T_{N}$ theories, JHEP 01 (2015) 093 [arXiv: 1409.0571] [INSPIRE].

[39] V. Mitev and E. Pomoni, Toda 3-point functions from topological strings, JHEP 06 (2015) 049 [arXiv: 1409.6313] [INSPIRE].

[40] O. Bergman and G. Zafrir, Lifting 4d dualities to 5d, JHEP 04 (2015) 141 [arXiv: 1410.2806] [INSPIRE].

[41] V. Mitev, E. Pomoni, M. Taki and F. Yagi, Fiber-base duality and global symmetry enhancement, JHEP 04 (2015) 052 [arXiv: 1411.2450] [INSPIRE].

[42] S.-S. Kim and F. Yagi, $5 d E_{n}$ Seiberg-Witten curve via toric-like diagram, JHEP 06 (2015) 082 [arXiv: 1411.7903] [INSPIRE].

[43] M. Isachenkov, V. Mitev and E. Pomoni, Toda 3-point functions from topological strings II, arXiv:1412.3395 [INSPIRE].

[44] Y. Tachikawa, Instanton operators and symmetry enhancement in 5d supersymmetric gauge theories, Prog. Theor. Exp. Phys. 2015 (2015) 043B06 [arXiv:1501.01031] [InSPIRE].

[45] G. Zafrir, Instanton operators and symmetry enhancement in 5d supersymmetric USp, SO and exceptional gauge theories, JHEP 07 (2015) 087 [arXiv: 1503.08136] [INSPIRE].

[46] H. Hayashi and G. Zoccarato, Topological vertex for Higgsed $5 d T_{N}$ theories, arXiv: 1505.00260 [INSPIRE].

[47] O. Aharony and A. Hanany, Branes, superpotentials and superconformal fixed points, Nucl. Phys. B 504 (1997) 239 [hep-th/9704170] [INSPIRE].

[48] O. Aharony, A. Hanany and B. Kol, Webs of $(p, q)$ five-branes, five-dimensional field theories and grid diagrams, JHEP 01 (1998) 002 [hep-th/9710116] [INSPIRE].

[49] F. Benini, S. Benvenuti and Y. Tachikawa, Webs of five-branes and $\mathcal{N}=2$ superconformal field theories, JHEP 09 (2009) 052 [arXiv:0906.0359] [INSPIRE].

[50] S.-S. Kim, M. Taki and F. Yagi, Tao probing the end of the world, arXiv:1504.03672 [INSPIRE].

[51] A. Hanany and A. Zaffaroni, Branes and six-dimensional supersymmetric theories, Nucl. Phys. B 529 (1998) 180 [hep-th/9712145] [INSPIRE].

[52] I. Brunner and A. Karch, Branes at orbifolds versus Hanany Witten in six-dimensions, JHEP 03 (1998) 003 [hep-th/9712143] [inSPIRE].

[53] N. Seiberg, Nontrivial fixed points of the renormalization group in six-dimensions, Phys. Lett. B 390 (1997) 169 [hep-th/9609161] [INSPIRE]. 
[54] U.H. Danielsson, G. Ferretti, J. Kalkkinen and P. Stjernberg, Notes on supersymmetric gauge theories in five-dimensions and six-dimensions, Phys. Lett. B 405 (1997) 265 [hep-th/9703098] [INSPIRE].

[55] M. Bershadsky and C. Vafa, Global anomalies and geometric engineering of critical theories in six-dimensions, hep-th/9703167 [INSPIRE].

[56] M.R. Douglas and G.W. Moore, D-branes, quivers and ALE instantons, hep-th/9603167 [INSPIRE].

[57] H. Hayashi, S.-S. Kim, K. Lee, M. Taki and F. Yagi, to appear.

[58] K. Yonekura, Instanton operators and symmetry enhancement in 5d supersymmetric quiver gauge theories, JHEP 07 (2015) 167 [arXiv:1505.04743] [INSPIRE].

[59] A. Sen, F theory and orientifolds, Nucl. Phys. B 475 (1996) 562 [hep-th/9605150] [INSPIRE].

[60] A. Hanany and E. Witten, Type IIB superstrings, BPS monopoles and three-dimensional gauge dynamics, Nucl. Phys. B 492 (1997) 152 [hep-th/9611230] [InSPIRE].

[61] H. Hayashi, Y. Tachikawa and K. Yonekura, Mass-deformed $T_{N}$ as a linear quiver, JHEP 02 (2015) 089 [arXiv: 1410.6868] [INSPIRE].

[62] L. Bao, E. Pomoni, M. Taki and F. Yagi, M5-branes, toric diagrams and gauge theory duality, JHEP 04 (2012) 105 [arXiv:1112.5228] [INSPIRE].

[63] K.A. Intriligator, D.R. Morrison and N. Seiberg, Five-dimensional supersymmetric gauge theories and degenerations of Calabi-Yau spaces, Nucl. Phys. B 497 (1997) 56 [hep-th/9702198] [INSPIRE].

[64] M.R. Gaberdiel and B. Zwiebach, Exceptional groups from open strings, Nucl. Phys. B 518 (1998) 151 [hep-th/9709013] [INSPIRE].

[65] M.R. Gaberdiel, T. Hauer and B. Zwiebach, Open string-string junction transitions, Nucl. Phys. B 525 (1998) 117 [hep-th/9801205] [INSPIRE]. 